\title{
IDENTIFICACIÓN DE FACTORES DE TIPO CATEGÓRICO RELACIONADOS CON LA CONDICIÓN ESTRUCTURAL DE TUBERÍAS DE ALCANTARILLADO DE BOGOTÁ A PARTIR DE CONCEPTOS DE ENTROPÍA DE LA INFORMACIÓN
}

\author{
Nathalie Hernández-Rodríguez ${ }^{1}$, Nelson Obregón-Neira² ${ }^{2}$ Andrés Torres ${ }^{3}$ \\ ${ }^{1}$ Estudiante de Maestría en Hidrosistemas, Grupo de Investigación Ciencia e Ingeniería del Agua y el Ambiente. \\ Correo electrónico: ingrid.hernandez@javeriana.edu.co \\ ${ }^{2}$ Doctor en Ciencias Hidrológicas, Director de Instituto Geofísico, profesor titular \\ ${ }^{3}$ Doctor en Hidrología Urbana, Grupo de Investigación Ciencia e Ingeniería del Agua y el Ambiente, Departamento \\ de Ingeniería Civil, profesor titular \\ Pontificia Universidad Javeriana, Bogotá, Colombia
}

Recibido: 21 de agosto del 2015 Aprobado: 10 de noviembre del 2015

Cómo citar este artículo: N. Hernández-Rodríguez, N. Obregón-Neira y A. Torres, "Identificación de factores de tipo categórico relacionados con la condición estructural de tuberías de alcantarillado en Bogotá a partir de conceptos de entropía de la información", Ingeniería Solidaría, vol. 12, n. ${ }^{0}$ 19, pp. 63-71, abril 2016, doi: http://dx.doi.org/10.16925/in.v12i19.1194

Resumen. Introducción: este artículo es producto del proyecto de investigación "Identificación de factores de tipo categórico con la condición estructural de tuberías de alcantarillado de Bogotá a partir de conceptos de entropía de la información”, financiado por la Maestría en Hidrosistemas de la Pontificia Universidad Javeriana, y desarrollado durante el 2014 y el 2015. El objetivo fue identificar qué influencia tienen factores de tipo cualitativo (tipo de red, material de la superficie vial asociada y material de las tuberías) sobre el grado estructural obtenido a partir de inspecciones CCTV realizadas en Bogotá entre el 2007 y el 2011. Metodología: se estudiaron los conceptos de entropía de la información mutua a fin de encontrar independencia entre las siguientes variables categóricas: tipo de tubería, tipo de superficie vial sobre la tubería y material de la tubería. Resultados: obtenidos a partir de la aplicación de conceptos tales como entropía e información mutua, muestran que la categoría estructural es independiente del tipo de tubería, del tipo de material de la tubería y del tipo de superficie de la vía que pasa sobre la tubería, ya que presentan valores elevados de entropía conjunta, lo cual implica un alto grado de incertidumbre en sus relaciones. Conclusiones: las variables categóricas estudiadas son factores importantes para tener en cuenta en un modelo de deterioro de las tuberías de alcantarillado, además de facilitar la toma de decisiones para direccionar los planes de inspección del alcantarillado en la ciudad.

Palabras clave: entropía de la información, gestión de activos, gestión proactiva, información mutua, redundancia, sistemas de alcantarillado. 


\title{
Factor Identification of Categorical Type Related to the Structural Condition of Bogota's Sewage Pipelines Stemming from CONCEPTS OF THE INFORMATION'S ENTROPY
}

\begin{abstract}
Introduction: this article is the result of the research project "Factor identification of categorical type related to the structural condition of Bogota's sewage pipelines stemming from concepts of information's entropy" financed by the Mastery in Hydro-systems of the Pontifical University Javeriana and developed in 2014 and 2015. The objective was to identify the influence of factors of qualitative type (grid type, material of the associated road surface and material of the pipeline) on the structural grade obtained from CCTV inspections in Bogota during 2007 and 2011. Methodology: the entropy's concept of mutual information was studied to differentiate the following categorical variables: type of pipelines, type of road surface over the pipeline and materials of the pipeline. Results: obtained from the application of concepts like entropy and mutual information show that the structural classification is independent from the type of pipeline, since they present elevated values of joined entropy and imply a high degree of uncertainty in their relation. Conclusions: the categorical variables studied are important factors to account in an impairment model of sewage pipelines; they also provide decision-making to direct the inspection plans of sewage in the city.
\end{abstract}

Keywords: entropy of information, file management, proactive management, mutual information, redundancy, sewage systems.

\section{IDENTIFICAÇÃO DE FATORES DE TIPO CATEGÓRICO RELACIONADOS COM A CONDIÇÃO ESTRUTURAL de TUbulaÇão de esgoto de Bogotá a PARTir DE CONCEITOS DE ENTROPIA DA INFORMAÇÃO}

Resumo. Introdução: este artigo é produto do projeto de investigação "Identificação de fatores de tipo categórico com a condição estrutural de tubulações de esgoto de Bogotá a partir de conceitos de entropia da informação", financiado pelo Mestrado em Hidrosistemas da Pontifícia Universidade Javeriana, e desenvolvido durante os anos 2014 e 2015. O escopo foi identificar a influência que tem fatores do tipo qualitativo (tipo de rede, material da superfície viaria associada e material das tubulações) sobre o grau estrutural obtido a partir de inspeções ССTV realizadas em Bogotá entre o ano 2007 e 2011. Metodologia: estudaram-se os conceitos de entropia da informação mutua para achar independência entre as seguintes variáveis categóricas: tipo de tubulação, tipo de superfície viaria sobre a tubulação e material da tubulação. Resultados: obtidos a partir da aplicação de conceitos tales como entropia e informação mutua, mostram que a categoria estrutural é independente do tipo de tubulação, do tipo de material da tubulação e do tipo de superfície da via que passa sobre a tubulação, já que apresentam valores elevados de entropia conjunta, envolvendo um alto grau de incerteza em suas relações. Conclusões: as variáveis categóricas estudadas são fatores importantes para levar em conta em um modelo de deterioração das tubulações de esgoto, além de facilitar a tomada de decisões para direcionar os planos de inspeção do esgoto na cidade.

Palavras-chave: entropia da informação, gestão de ativos, gestão proativa, informação mutua, redundância, sistemas de esgoto. 


\section{Introducción}

Como consecuencia del crecimiento demográfico en las ciudades, los hidrosistemas urbanos se encuentran sometidos a presiones cada vez mayores en términos de cambio climático, contaminación ambiental, limitación de recursos y envejecimiento de la infraestructura [1]. Dentro de la infraestructura desarrollada por las ciudades a través de los años se encuentran las redes de drenaje. Según reportes provenientes de países desarrollados y en vías de desarrollo, esta infraestructura presenta tasas alarmantes de envejecimiento y deterioro [2]. Es así como la mayoría de redes de alcantarillado son cada vez más propensas a fallar [3], además de traer como consecuencia su deterioro estructural, hidráulico e impactando el nivel de servicio y, por consiguiente, la calidad de vida de las comunidades $[2,4,5]$.

Existen múltiples factores que intervienen en el deterioro de las tuberías, tales como las características físicas (diámetro, longitud, profundidad, material, tipo de unión); los procesos constructivos de instalación; factores externos (las características del suelo de soporte, uso del suelo y características ambientales); y otros factores como la edad de la tubería, métodos inapropiados de mantenimiento y el tipo de tubería utilizado [6]. En la literatura especializada, se mencionan también factores sociales, económicos y ambientales tales como el crecimiento demográfico, el desarrollo de la ciudad y el cambio climático [7]. Adicionalmente, se debe tener en cuenta que los procesos de deterioro de las tuberías de aguas residuales y lluvias son diferentes: las tuberías de aguas residuales están sujetas a ataques internos por ácidos, mientras que las tuberías de aguas lluvias están sujetas a factores externos [4]. Por otra parte, las redes subterráneas de servicios urbanos se consideran sistemas complejos por la intervención e interacción de los factores anteriormente mencionados [8]. Sin embargo, la intervención e interacción de estos factores no han sido significativamente estudiados [9].

A nivel global, han surgido algunas metodologías para el pronóstico de la condición estructural de las tuberías de alcantarillado. Dichas metodologías se basan en diversas herramientas como cadenas de Márkov [10], regresiones simples [11], estadística bayesiana [4], regresiones ordinales, regresiones logísticas multinomiales y binarias [12], así como metodologías de aprendizaje de máquina tales como redes neuronales artificiales y máquina de soporte vectorial [13].

Sin embargo, a pesar de que en el ámbito internacional existe una diversidad de modelos que permiten pronosticar la condición estructural de las tuberías, dichos modelos se basan en condiciones estructurales obtenidas mediante normas municipales, nacionales $\mathrm{e}$ internacionales, a partir de un análisis exhaustivo de los resultados de inspecciones realizadas mediante circuito cerrado de televisión (CCTV), sensores electrónicos o sistemas de sonido (DTs-digital theater systems), entre otros $[9,8,14,15]$. Además, la mayoría de estos modelos dependen de variables de entradas verídicas y completas cuya información resulta limitada, considerando la escasez (baja cobertura de inspecciones de la red), y la calidad de la información, ya que este tipo de tecnologías (CСTV) es utilizada por varios contratistas externos de la empresa prestadora de este servicio público [16].

En Bogotá, en el 2001, se emitió la norma Ns-058 [17] con el fin de evaluar las redes inspeccionadas mediante CCTV. Con base en dichas evaluaciones de tramos aislados, se tienen en cuenta aspectos estructurales y operacionales. A partir de ese puntaje, se dan indicaciones para tomar decisiones en cuanto a rehabilitación y acciones preventivas de los alcantarillados inspeccionados. Asimismo, se han desarrollado estudios de identificación de factores que intervienen en los riesgos físicos, ambientales y operacionales en las redes de drenaje de Bogotá, a partir de recopilaciones de inspecciones del CCTV y la información de características físicas de las tuberías. Uno de ellos estableció cómo el material y diámetro de las tuberías presentaban una correlación significativa con la magnitud y ocurrencia de fallos, sobre todo, en materiales como Gres y PVC, y diámetros menores a 20 pulgadas. Por otro lado, el tipo de superficie vial asociada (pavimentada, sin pavimentar o verde), y el tipo de red (sanitaria, pluvial, combinada), están significativamente relacionados solamente con el tipo de falla estructural [18]. Sin embargo, este estudio se realizó por medio de un análisis de regresión lineal simple, dejando a un lado el hecho de que factores como el material de las tuberías, el tipo de red y el tipo de superficie vial no son cuantificables numéricamente y, por lo tanto, se debe identificar su relación, la cual puede ser de tipo no lineal, con la condición por medio de métodos que contemplen variables categóricas.

Por otro lado, existen otros autores que en sus estudios identifican características físicas de las tuberías como factores que influyen de manera importante sobre la condición operativa de las redes de drenaje urbano, al relacionarlas con confiabilidades de inundación y operación de las tuberías de la red perteneciente al alcantarillado urbano $[19,20]$. 
Dado lo anterior, se percibe que el reconocimiento de factores que inciden sobre el deterioro de las tuberías de drenaje urbano, y en particular causado por daños estructurales, es un primer acercamiento para desarrollar herramientas que soporten la gestión de mantenimiento proactivo de las tuberías de alcantarillado en Bogotá. Adicionalmente, se reconoce como factores clave que pueden tener una incidencia importante sobre el estado estructural de las tuberías de alcantarillado, son de tipo cualitativo $\mathrm{y}$, por lo tanto, son menos adaptados a análisis por medio de métodos de estadística lineal. Es por esto que en este artículo se pretende conocer la influencia de los factores de tipo cualitativo encontrados en la literatura (tipo de red, tipo de material y tipo de vía), sobre el grado estructural por medio del concepto de la entropía y más específicamente con el concepto de información mutua (MI).

\section{Materiales y métodos}

Para la evaluación de la condición estructural de las tuberías de la red de alcantarillado de Bogotá, se contó con una tercera parte de la base de datos de inspecciones por CCTV realizadas entre el $2007 \mathrm{y}$ el 2011, por parte de la empresa prestadora del servicio público de agua y alcantarillado de Bogotá, Empresa de Acueducto y Alcantarillado de Bogotá (EAB; figura 1). Dicha base de datos contiene información de las características físicas de las tuberías, su localización, el puntaje y grado estructural producto de estas inspecciones obtenidas con base en la norma Ns-058 [17]. La base de datos contiene 3563 inspecciones de tuberías pluviales y sanitarias (locales y troncales).

Los resultados de las inspecciones de CCTV vienen dados a partir de la norma Ns-058 de la Empresa de Acueducto de Bogotá (ЕAB), en la cual, a partir de la magnitud, la gravedad y el daño o daños encontrados en las tuberías de alcantarillado, cada tubería recibe un puntaje estructural. La norma establece una reagrupación de dicho puntaje a partir de su criticidad para facilitar una clasificación de la condición estructural, por lo tanto, parametriza este puntaje en cinco grados estructurales, los cuales tienen cinco descripciones de la condición estructural de las tuberías tal como se observa en la tabla 2.
Con el fin de simplificar y tomar decisiones más eficientes, se agruparon en tres categorías de estos grados estructurales. Categoría 1: grado 1; Categoría 2: grado 2, 3, 4; y Categoría 3: grado 5.

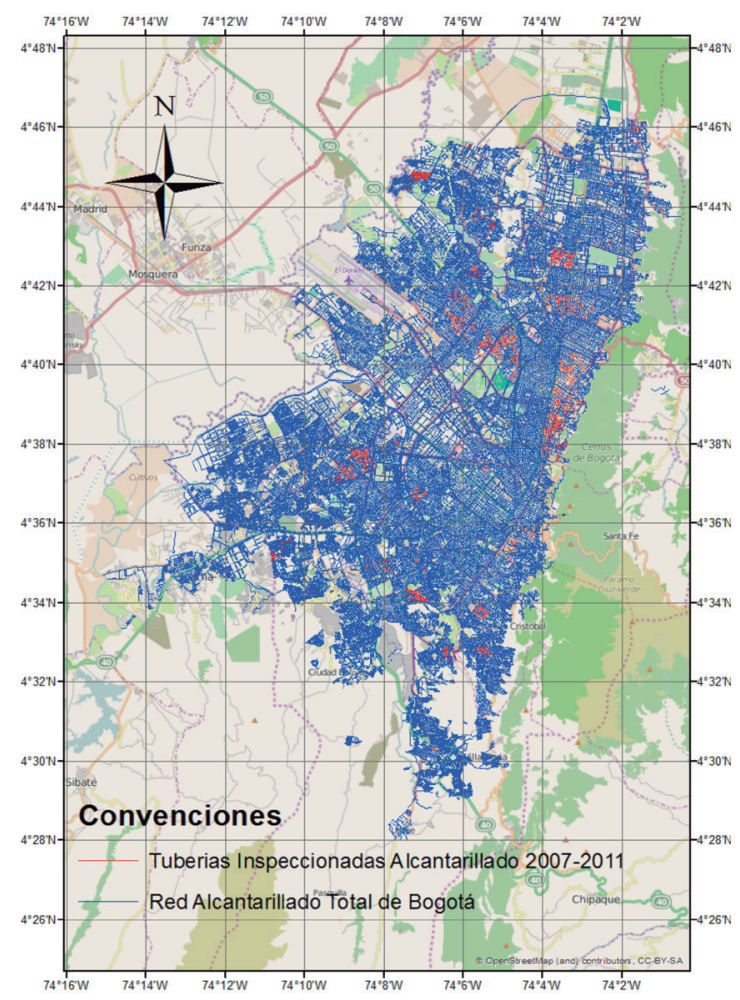

Figura 1. Mapa de tuberías inspeccionadas durante el periodo 2007-2011 y de la red de alcantarillado en Bogotá D.C.

Fuente: elaboración propia

Tabla 1. Variables de tipo cualitativo y sus elementos categóricos

\begin{tabular}{|l|l|}
\hline \multicolumn{1}{|c|}{ Variable } & \multicolumn{1}{c|}{ Elementos } \\
\hline $\begin{array}{l}\text { Tipo } \\
\text { tubería }\end{array}$ & $\begin{array}{l}\text { Sanitaria, Pluvial, Sanitaria_Troncal, } \\
\text { Pluvial_Troncal }\end{array}$ \\
\hline Material & Concreto, Gres, Pvc y Mampostería \\
\hline Tipo vía & $\begin{array}{l}\text { Pavimentada en concreto, pavimentada } \\
\text { en asfalto, superficie verde, superficie } \\
\text { afirmada }\end{array}$ \\
\hline
\end{tabular}

Fuente: elaboración propia 
Tabla 2. Determinación del grado estructural a partir del puntaje estructural y su descripción

\begin{tabular}{|l|c|l|}
\hline \multicolumn{1}{|c|}{ Puntaje } & Grado & \multicolumn{1}{c|}{ Descripción } \\
\hline $\begin{array}{l}\text { Menos de } \\
10\end{array}$ & 1 & Sin defectos estructurales \\
\hline $10-39$ & 2 & Defectos de menor importancia \\
\hline $40-79$ & 3 & $\begin{array}{l}\text { Defectos que pueden generar } \\
\text { problemas estructurales e } \\
\text { hidráulicos }\end{array}$ \\
\hline $80-164$ & 4 & $\begin{array}{l}\text { Defectos de gran importancia, } \\
\text { tomar medidas preventivas y/o } \\
\text { correctivas }\end{array}$ \\
\hline $165+$ & 5 & $\begin{array}{l}\text { Tubería colapsada o cerca de } \\
\text { hacerlo, tomar medidas de } \\
\text { emergencia }\end{array}$ \\
\hline
\end{tabular}

Fuente: elaboración propia

De la base de datos descrita en la tabla 1, se tomaron las variables de tipo cualitativo (tipo de tubería, tipo de superficie vial asociada y material de tubería), así como el grado estructural otorgado a cada tubería. A esta nueva base de datos, se aplicaron los conceptos de entropía de la información [21] y de información mutua [22], con el propósito de establecer si se trata de variables independientes a las categorías planteadas que reúnen los grados estructurales otorgados por la norma NS-058 del 2001 [17].

En los siguientes diagramas de barras expuestos en la figura 2, se muestra una descripción de los elementos existentes en cada variable categórica de la base de datos. Como se observa, más del $60 \%$ de las tuberías inspeccionadas son tuberías sanitarias locales, le siguen las tuberías pluviales locales con un $30 \%$, las pertenecientes a la red troncal pluvial fueron de un $7 \% \mathrm{y}$, por último, las menos inspeccionados fueron las tuberías pertenecientes a la red troncal sanitaria con un $2 \%$ de todas las tuberías inspeccionadas entre el 2007 y el 2011 en Bogotá (ver figura 2a). Como se observa en la figura 2b, más del $60 \%$ de las tuberías inspeccionadas están debajo de una vía pavimentada en asfalto, le sigue con un 17\% las vías pavimentadas en concreto, luego afirmadas pero no pavimentadas y, por último, superficies verdes (superficies naturales). Se observa además que más del $80 \%$ de las tuberías son de gres y concreto en proporciones casi iguales de $40 \%$, y los materiales que menos se encontraron fueron aquellas tuberías en PVC (ver figura 2c). Finalmente, en la figura 2d, se observa que las tuberías inspeccionadas presentan las tres categorías de manera cuasi-equitativa, un $60 \%$ de las tuberías de alcantarillado en categorías 1 y 3 con una frecuencia equitativa de $30 \%$ en estas dos condiciones, y la categoría 2 en un $40 \%$ de las tuberías de alcantarillado inspeccionadas.

a)

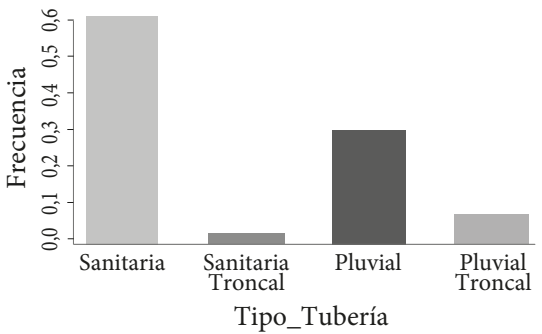

b)

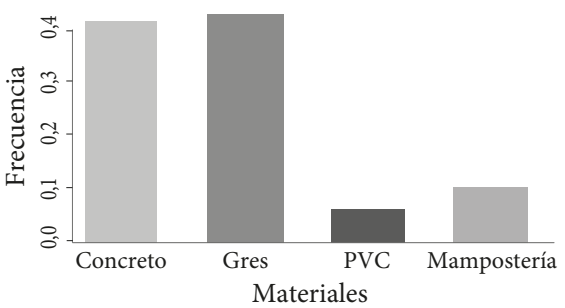

c)

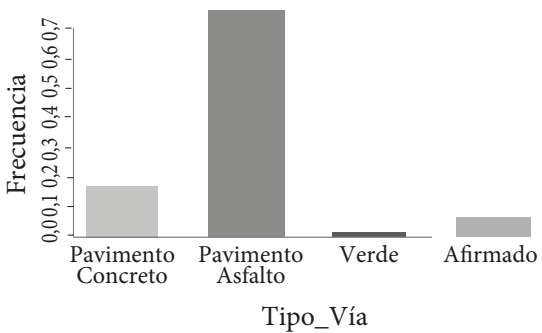

d)

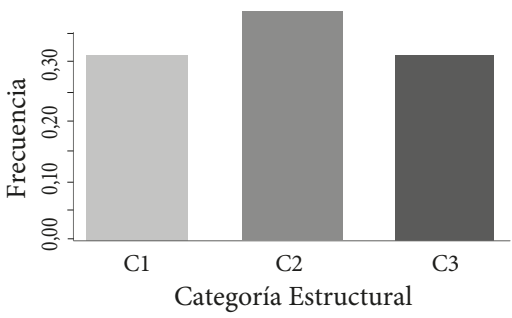

Figura 2. Diagramas de barras a) frecuencia tipo de tuberías inspeccionadas; b) frecuencia material de tuberías inspeccionadas; c) frecuencia tipo de vía por encima de las tuberías inspeccionadas; d) frecuencia categorías estructurales de las tuberías inspeccionadas Fuente: elaboración propia 


\section{Resultados}

En la tabla 3, se muestra la entropía de información de cada variable, y se observa como la variable que tiene mayor entropía es el material y la categoría estructural, es decir, variables que tienen mayor incertidumbre, lo cual implica mayor aleatoriedad. Por otro lado, la variable que tiene menor incertidumbre es el tipo de vía, lo cual significa que el tipo de vía tiene mayor cantidad de información estructurada.

Tabla 3. Entropía de las variables categóricas

\begin{tabular}{|l|c|}
\hline Variables & Entropía \\
\hline Tipo tubería & 1,32 \\
\hline Vía & 1,03 \\
\hline Material & 1,605 \\
\hline Categoría estructural & 1,58 \\
\hline
\end{tabular}

Fuente: elaboración propia

En la tabla 4, se observa la entropía conjunta para todas las posibles relaciones que se pueden hacer entre las variables categóricas: se observa que el material y la categoría estructural son el par que presentan mayor incertidumbre entre sus elementos. Por el contrario, la entropía conjunta de las variables tipo de vía y categoría estructural es la menor, lo cual implica que estas dos variables comparten mayor información.

Tabla 4. Entropía conjunta de las variables categóricas

\begin{tabular}{|l|l|c|}
\hline \multicolumn{1}{|c|}{ Variable 1 } & \multicolumn{1}{|c|}{ Variable 2 } & $\begin{array}{c}\text { Entropía } \\
\text { Conjunta }\end{array}$ \\
\hline Tipo tubería & Categoría estructural & 2,81 \\
\hline Material & Categoría estructural & 3,0054 \\
\hline Vía & Categoría estructural & 2,59 \\
\hline
\end{tabular}

Fuente: elaboración propia

En la tabla 5, se observan los valores de información mutua por pares de variables, en los cuales se da una indicación de la cantidad de información que contiene una variable aleatoria con respecto a otra. Lo anterior da una idea de la independencia o dependencia entre las variables categóricas con respecto al grado estructural. En este estudio, se encontró que el par tipo de vía y el grado estructural es la relación que presenta mayor dependencia debido a que su cantidad de información es mayor.

Tabla 5. Información mutua entre pares de variables categóricas

\begin{tabular}{|l|l|c|}
\hline \multicolumn{1}{|c|}{ Variable 1 } & Variable 2 & $\begin{array}{c}\text { Información } \\
\text { mutua }\end{array}$ \\
\hline Tipo tubería & Categoría estructural & 0,089 \\
\hline Material & Categoría estructural & 0,18 \\
\hline Vía & Categoría estructural & 0,32 \\
\hline
\end{tabular}

Fuente: elaboración propia

En la tabla 6, se encuentran los coeficientes de incertidumbre por cada par de variables. Este coeficiente de incertidumbre mide la reducción proporcional del error cuando los valores de una variable se emplean para predecir valores de la otra. Si el coeficiente es próximo a 1 , significa que las variables independientes (tipo de tubería, material y tipo de vía), pronostican perfectamente la variable dependiente (categoría estructural).

Tabla 6. Coeficientes de Incertidumbre (Cyx)

\begin{tabular}{|l|l|c|}
\hline $\begin{array}{c}\text { Variable In- } \\
\text { dependiente }\end{array}$ & $\begin{array}{c}\text { Variable } \\
\text { dependiente }\end{array}$ & $\begin{array}{c}\text { Coeficiente de } \\
\text { Incertidumbre }\end{array}$ \\
\hline Tipo tubería & Categoría estructural & 0,067 \\
\hline Material & Categoría estructural & 0,11 \\
\hline Vía & Categoría estructural & 0,31 \\
\hline
\end{tabular}

Fuente: elaboración propia

En la tabla 7, se presenta el coeficiente de redundancia de cada variable categórica con respecto a la categoría estructural. La idea con este coeficiente es conocer la capacidad predictiva de esta variable frente a la categoría estructural. Si el coeficiente de redundancia tiende a cero, no existe redundancia y, por lo tanto, las variables analizadas en cada par son independientes. A fin de conocer qué tan alta puede ser su redundancia se halla su máximo valor. Al comparar los resultados obtenidos (ver tabla 7), se observa que las variables categóricas analizadas son independientes de las categorías estructurales con porcentajes de redundancia máximo del $31 \%$, aproximadamente, en el tipo de vía y la categoría estructural. 
Tabla 7. Coeficiente de redundancia (R), Redundancia máxima y Porcentaje de redundancia

\begin{tabular}{|l|l|c|c|c|}
\hline $\begin{array}{c}\text { Varia- } \\
\text { ble 1 }\end{array}$ & $\begin{array}{c}\text { Varia- } \\
\text { ble 2 }\end{array}$ & $\begin{array}{c}\text { Redun- } \\
\text { dancia }\end{array}$ & $\begin{array}{c}\text { Redun- } \\
\text { dancia } \\
\text { máxima }\end{array}$ & $\begin{array}{c}\% \text { Re- } \\
\text { dundan- } \\
\text { cia }\end{array}$ \\
\hline $\begin{array}{l}\text { Tipo } \\
\text { tubería }\end{array}$ & $\begin{array}{l}\text { Categoría } \\
\text { estructu- } \\
\text { ral }\end{array}$ & 0,031 & 0,46 & 7 \\
\hline Material & $\begin{array}{l}\text { Categoría } \\
\text { estructu- } \\
\text { ral }\end{array}$ & 0,056 & 0,5 & 11 \\
\hline Vía & $\begin{array}{l}\text { Categoría } \\
\text { estructu- } \\
\text { ral }\end{array}$ & 0,12 & 0,39 & 31 \\
\hline
\end{tabular}

Fuente: elaboración propia

Debido a los anteriores resultados, fue pertinente encontrar otro método que relacionara las variables categóricas con las categorías de la condición estructural. Por lo tanto, se realizó un análisis de varianzas no paramétrico (Kruskal-Wallis). Los resultados obtenidos indican que, tanto el tipo de tubería, como el material y el tipo de vía, presentan una influencia significativa sobre la categoría estructural (ver tabla 8 ).

Tabla 8. P-value de la prueba Kruskal-Wallis

\begin{tabular}{|l|l|l|}
\hline \multicolumn{1}{|c|}{ Variable 1 } & \multicolumn{1}{c|}{ Variable 2 } & \multicolumn{1}{c|}{ P-value } \\
\hline Tipo tubería & Categoría estructural & $2.20 \mathrm{E}-16$ \\
\hline Material & Categoría estructural & $2.20 \mathrm{E}-16$ \\
\hline Vía & Categoría estructural & $3.38 \mathrm{E}-15$ \\
\hline
\end{tabular}

Fuente: elaboración propia

\section{Discusión}

Los valores de información mutua y coeficiente de incertidumbre encontrados en este trabajo reflejan ser muy bajos si se comparan con su estandarización teórica (1: dependencia; 0: independencia) [22]. Sin embargo, estas variables presentan porcentajes de redundancia no insignificantes $(>5 \%)$ : (i) $31 \%$ para el tipo de superficie sobre la tubería; (ii) $11 \%$ para el material de la tubería; y (iii) $7 \%$ para el tipo de tubería. Lo anterior resulta satisfactorio para este trabajo, ya que indica cómo estas variables categóricas son factores a tener en cuenta en modelos de deterioro de tuberías de alcantarillado, además de facilitar la toma de decisiones en el propósito de direccionar los planes de inspección del alcantarillado en la ciudad.

Por otro lado, se corroboró que el tipo de tubería, el tipo de material y el tipo de superficie de la vía tienen una influencia significativa sobre la condición estructural de las tuberías de alcantarillado representada en tres categorías, según la prueba KruskalWallis. Estos resultados son acordes con aquellos presentados por [18], en los cuales posiciona el material como factor de riesgo de la condición estructural de las tuberías cuando se relaciona con la gravedad de la falla y la cantidad de fallos (índices que componen la calificación de la condición estructural de las tuberías de alcantarillado según la Norma Ns-058 de la ЕАВ), y afirma que el tipo de superficie de la vía y el tipo de tubería no solo tienen relación con el tipo de falla, sino con la condición estructural de las tuberías de alcantarillado representada en categorías estructurales en el presente trabajo.

Por otro lado, en un trabajo desarrollado por Torres et al. [19], se realizó esta misma prueba estadística (Kruskal-Wallis), con el fin de determinar la influencia de las características físicas de las tuberías de alcantarillado sobre la confiabilidad de inundación y operación del sistema de alcantarillado en una cuenca (Salitre) de Bogotá. En sus resultados, el material tiene una influencia significativa con respecto a la confiabilidad de inundación. Del mismo modo, Torres et al. [19] encontraron que el tipo de tubería presenta una influencia significativa en la confiabilidad de operación (probabilidad de que un producto pueda realizar su función sin incidentes durante un periodo, bajo condiciones controladas) del sistema de alcantarillado. Posteriormente, los autores de [20] confirman al material como un factor que influye en la confiabilidad de inundación utilizando la información mutua como herramienta de evaluación. El valor de información mutua del material (representado por la rugosidad del material) y la confiabilidad de inundación son del orden de 0,7.

Del mismo modo, se compararon los anteriores resultados considerados locales (porque fueron desarrollados con bases de datos de alcantarillado de Bogotá), con aquellos de otros países: en el Reino Unido, se contemplan el material y el tipo de tubería como factores influyentes en el deterioro del sistema (corrosión). Asimismo, el tipo de superficie también es considerado factor influyente en el deterioro de las tuberías, toda vez que este factor está ligado con el tipo de tráfico que transita sobre ellas [6]. 
En resumen, el tipo y el material de la tubería, así como el tipo de superficie vial, son factores que influyen de manera significativa en el deterioro de las tuberías.

\section{Conclusiones}

Los resultados obtenidos a partir de la aplicación de conceptos de la entropía e información mutua en este trabajo muestran cómo la categoría estructural es independiente del tipo de tubería, del tipo de material de la tubería y del tipo de superficie de la vía que pasa sobre la tubería, ya que presentan valores elevados de entropía conjunta, lo cual implica un alto grado de incertidumbre en sus relaciones. Además, los valores de información mutua y coeficiente de incertidumbre son muy bajos, lo cual refleja la baja capacidad de predicción de estas variables frente a la categoría estructural. Sin embargo, estas variables presentan porcentajes de redundancia no insignificantes (>5\%): (i) $31 \%$ para el tipo de superficie sobre la tubería; (ii) $11 \%$ para el material de la tubería; y (iii) $7 \%$ para el tipo de tubería. Lo anterior resulta satisfactorio para este trabajo, ya que indica que estas variables categóricas son factores importantes para tener en cuenta en un modelo de deterioro de las tuberías de alcantarillado, además de facilitar la toma de decisiones a fin de direccionar los planes de inspección del alcantarillado en la ciudad.

Por otro lado, este trabajo complementa los hallazgos obtenidos por [18], [19] y [20], al corroborar que el tipo de tubería, el tipo de material y el tipo de superficie de la vía tienen una influencia significativa sobre la condición estructural de las tuberías de alcantarillado representada en tres categorías, según la prueba Kruskal-Wallis.

\section{Agradecimientos}

Los autores agradecen a la EAB por la información suministrada para la realización de la presente investigación.

\section{Referencias}

[1] B. C. Ferguson, R. R. Brown y A. Deletic, "Diagnosing transformative change in urban water systems: Theories and frameworks, Global Environmental Change", vol. 23, n. ${ }^{\circ}$ 1, feb. 2013, pp. 264-280.
[2] H. Osman, "Agent-based simulation of urban infrastructure asset management activities, Automation in Construction", vol. 28, pp. 45-57, dic. 2012

[3] B. Ward y D. A. Savić, "A multi-objective optimisation model for sewer rehabilitation considering critical risk of failure", Water Sci Technol, vol. 66, n. ${ }^{\circ}$ 11, pp. 2410-2417, 2012. doi: 10.2166/wst.2012.393

[4] T. Micevski, G. Kuczera y P. Coombes, "Markov Model for Storm Water Pipe Deterioration," J. Infrastruct. Syst., vol. 8, n. ${ }^{\circ}$ 2, pp. 49-56, jun. 2002. doi: http://dx. doi.org/10.1061/(ASCE)1076-0342(2002)8:2(49)

[5] Z. Liu y Y. Kleiner, "State of the art review of inspection technologies for condition assessment of water pipes", Measurement, vol. 46, n. ${ }^{\circ} 1$, ene. 2013, pp. 1-15. doi: http://dx.doi.org/10.1016/j.measurement.2012.05.032

[6] J. P. Davis, B. A. Clarke, J. T. Whiter y R. J Cunninghan, "Factors influencing the structural deterioration and collage of rigid sewer pipes", Urban water, vol. 3, n. ${ }^{\circ}$ 1, pp. 73-89, 2001. doi: http://dx.doi. org/10.1016/ S1462-0758(01)00017-6

[7] M. Kleidorfer, M. Moderl, F. Tscheirker-Gratl, M. Hammerer, H. Kinzel y Rauch W, "Integrated planning of rehabilitation strategies for sewers", Water Science \& Technology, vol. 68, n. ${ }^{\circ}$ 1, pp. 173-183, 2013. doi: 10.2166

[8] T. Hao et al., "Condition assesment of the buried utility service infraestructure", Tunneling and Underground Space technology, n. ${ }^{\circ} 28$, pp. 331-344, 2012.

[9] J. Lee, Y. Jeong Y-S. Oh, J-C. Lee, N. Ahn, J. Lee y $\mathrm{S}-\mathrm{H}$. Yoon, "An integrated approach to intelligent urban facilities management for real-time emergency response", Automation in Construction, vol. 30, pp. 256-264, mar. 2013. doi: http://dx.doi.org/10.1016/j.autcon.2012.11.008

[10] H-S. Baik, H. S. D. Jeong y D. M. Abraham, "Estimating Transition Probabilities in Markov Chain-Based Deterioration Models for Management of Wastewater Systems", J. Water Resour. Plann. Manage, vol. 132, n. ${ }^{\circ} 1$, pp. 15-24, ene. 2006. doi: http://dx.doi. org/10.1061/(ASCE)0733-9496 (2006)132:1(15)

[11] R. Wirahadikusumah, D. Abraham y T. Iseley, "Challenging issues in modelling deterioration of combined sewers" Journal of Infrastructure Systems, vol. 7, n. ${ }^{\circ}$, pp. 77-84, jun. 2001. doi: http://dx.doi.org/10.1061/ (ASCE)1076-0342(2001)7:2(77)

[12] B. Salman y O. Salem, "Modeling failure of wastewater collection lines using various section-level regression models", Journal of Infrastructure Systems, vol. 18, n. ${ }^{\circ}$ 2, pp. 146-154. doi: http://dx.doi.org/10.1061/ (ASCE)IS.1943-555X.0000075

[13] J. Mashford, D. Marlow, D. Tran y R. May, "Prediction of Sewer Condition Grade Using Support 
Vector Machines", Journal of Computing in Civil Engineering, vol. 25, n. ${ }^{\circ}$ 4, pp. 283-290, jul. 2011.

[14] P. Davis, I. Martin, R. Gopirajy y J. Hicks, "Review of condition assessment techniques and decision frameworks for gravity sewers", $8^{\text {th }}$ International Conference on Sewer Process and Networks Surfers Paradise, Gold Coast, Australia, 7-10 nov., 2010.

[15] R. Schilperoort, E. Rudelle, J. Vollertsen, H. Hoppe, A. Lausten, U. Laschet, M. Uggerby y J. Langeveld, “CCTV vs. DTs: Comparison of Results in the Search for Infiltration and Inflow", $13^{\text {th }}$ International Conference of Urban Drainage, Sarawak, Mlaysia, 7-12 sep. 2014.

[16] J. P. Rodríguez, N. McIntyre, M. Díaz-Granados, J. P. Quijano, y Č. Maksimović, "Monitoring and modelling to support wastewater system management in developing mega-cities", Science of the Total Environment, vol. 445, pp. 79-93, feb. 2013. doi: http:// dx.doi.org/10.1016/ j.scitotenv.2012.12.022

[17] Empresa de Acueducto y Alcantarillado de Bogotá (EAAB). "NS - 058. Aspectos Técnicos para inspección y mantenimiento de redes y estructuras de alcantarillado", Bogotá, Colombia, EAAB-E.S.P., 2001.
[18] P. Niño, H. Angarita, D. Vargas y A. Torres, "Identificación factores de riesgo para la gestión patrimonial óptima de sistemas de drenaje urbano: Estudio Piloto en la Ciudad de Bogotá", xxv Congreso Latinoamericano de Hidráulica, San José, Costa Rica, 9-12 sep. 2012.

[19] A. Torres, S. Sandoval, K. Navarro y L. Pulido, "Strategies of maintenance in storm sewers in Bogotá Colombia by using estimates of operational and flooding reliabilities", en Proceedings of the 12th International Conference on Urban Drainage, Porto Alegre, Brazil, sept. 2011, p. 9.

[20] S. Sandoval, A. Torres, y N. Obregón, "Herramientas para la implementación de mantenimiento proactivo en alcantarillados urbanos utilizando confiabilidad de inundación y conceptos de entropía de información", Rev. Facultad de Ingeniería Universidad de Antioquia, n. ${ }^{\circ}$ 65, pp.152-166, 2012.

[21] R. M. Gray, Entropy and information theory. Stanford, USA: Springer Science \& Business Media, 2011. doi: http://dx.doi.org/10.1007/978-1-4419-7970-4

[22] T. M. Cover y J. A. Thomas, Elements of information theory, New Jersey, USA: John Wiley \& Sons, 2012. 